\title{
Capability and Uncertainty of Standardless Procedures for Quantitative Electron Probe X-ray Microanalysis
}

\author{
C. Merlet
}

\author{
ISTEEM, CNRS, Université de Montpellier II, Pl. E. Bataillon, 34095 Montpellier cedex 5, France
}

\begin{abstract}
Electron probe microanalysis (EPMA) has become a well established technique for determining compositions of bulk and multilayer samples. Quantification is traditionally based on the use of standards and many uncertainties, resulting of the experimental conditions, the atomic data and the physical model describing the X-ray emission, can be canceled. Depending of the instrument, the operator experience and the nature of the sample, the uncertainty in the results by the use of standards can be expected to be less than $2 \%$, even when mass absorption coefficient is very large. Unfortunately, measurements on many standards are time consuming and require the appropriate standard for the corresponding element. As a solution, standardless procedure [1-5] is an elegant way to overcome these obstacles. This method attempts to remove the need of standardization through calculation of pure element intensities. Two standardless procedures are largely used. The first one, based on absolute calculations, takes into account all aspects of X-ray generation, propagation, and detection, and the second one, based on mathematical fits, makes use of measured intensities from a limited set of pure standards. Since standardless procedures imply a decrease in accuracy, the aim of this paper is to evaluate the capability and the uncertainty of standardless analysis based on absolute quantification procedure, putting the emphasis on fundamental aspects.
\end{abstract}

To get reliable results, absolute quantification procedure requires three main points: - Firstly, an absolute quantification procedure needs an accurate description of the X-ray depth distribution from which the emitted X-ray intensities are calculated. X-ray depth distribution can be estimated by two different methods: Monte Carlo simulation [6,7] and analytical approximations [4,8]. Monte Carlo simulation, although more accurate, is very time consuming, even with the fastest computers available. Moreover, quantitative results are obtained with the help of automatic iterative numerical procedures and consequently, for on line quantification only analytical models are used in practice. Secondly, an absolute quantification procedure requires the accurate knowledge of atomic parameters that describe the electron interaction and the X-ray emission, such as the ionization cross section, mass absorption coefficient, fluorescent yields, Coster-Kronig transition and the relative transition probability (by using standards in quantitative microanalysis, many of these atomic parameters are canceled or do not need to be known accurately).- Thirdly, an absolute quantification procedure needs accurate determination of the detector efficiency. Although the long term stability of WDS instrument has been improved considerably, and standarless procedures with WDS instruments have been developed $[3,5]$, standarless analysis remains mainly the method of EDS systems [1-4] 
Apart from uncertainty in measurement, sources of errors in standarless procedure are principally the result from the atomic parameters and the detector efficiency. Nowadays, the accuracy in description of X-ray depth distribution is largely superior than the two previous points $[4,8]$.

Examination of the errors in standarless procedure reveals that the sources of the most significant errors are the atomic parameters. One of the main atomic parameters is the ionization cross section [1-4]. For this parameter, the common practice is to use semi-empirical formulas, which are affected by differences up to $40 \%$. Fortunately for the ionization cross section, recent experimental measurements and theoretical models based on the distorted-wave Born approximation (DWBA), show that we can now obtain uncertainty lower than $10 \%$ for $\mathrm{K}$ and L Lines [9-11]. In the case of analysis using $\mathrm{L}$ and $\mathrm{M}$ lines, the enhancement of intensity by Coster-Kronig transitions has to be taken into account. The sum of uncertainty on Coster-Kronig transitions is about $10 \%$ and the enhancement of intensity is at the maximum up to $40 \%$.

The second main source of errors in standarless procedure is the detector efficiency. It is important that the procedure contains a routine to calibrate the efficiency of the detector (WDS or EDS). Each type of detector needs calibration, and an efficient method is to use the background modeling in conjunction with the detector characteristic given by the manufacturer [9]

Right now, in most case involving $\mathrm{K}$ lines and for energies lines greater than $1 \mathrm{KeV}$, it is possible to obtain quite good results with standarless procedure. For the L and M lines the situation is less favorable, because of the uncertainty in radiationless transitions and the lack of accuracy of fluorescent yields. Despite the large errors generated in absolute quantification procedure for L and $\mathrm{M}$ lines and at low energies lines, it is worth to point out, that this method is attractive in many features, even for classical standard procedure with WDS instruments. There are some cases where standards are not available (rare gases, instability under irradiation or radioactive elements), and for such problems, absolute standard calculation is an elegant solution. In addition, spectrum simulation by using absolute emission calculation for $\mathrm{K}, \mathrm{L}$ and $\mathrm{M}$ lines is a very interesting tool to predict the feasibility, the detection limit, the precision or to choose the optimum experimental condition for a specific sample $[12,13]$

\section{References}

[1] D.E. Newbury et al., Anal. Chem. 67 (1995), 1866

[2] J. Labar, Microbeam Analysis, 4 (1995) 65

[3] J. Wernisch and K. Röhrbacher, Mikrochimica Acta., [Suppl.] 15, (1998) 307

[4] J.L. Pouchou, Mikrochimica Acta., 1114/115, (1994) 33

[5] C. Fournier, J. Anal. At. Spectrom., 14, (1999) 381.

[6] J. Baro et al., Nucl. Instr. And Meth. B, 100 (1995) 31

[7] R. Gauvin and E. Lifshin, Microscopy and Microanalysis, Vol 8, supp.2, (2002) 428.

[8] C. Merlet, Mikrochimica Acta, 114/115, (1994) 363

[9] X. Llovet et al., J. Phys. B: Atom. Mol. Opt. Phys., 33, (2000) 3761

[10] X. Llovet et al.. Mikrochimica Acta., 132, (2000), 163

[11] X. Llovet et al., J. Phys. B: Atom. Mol. Opt. Phys 35, (2002) 973.

[12] P. Duncumb, et al. Microscopy and Microanalysis, 7, 04 (2001) 341

[13] C. Fournier,. Mikrochimica Acta., 132, p. 531-539 